\title{
Cannabis-Based Medicines and Medical Cannabis for Chronic Neuropathic Pain
}

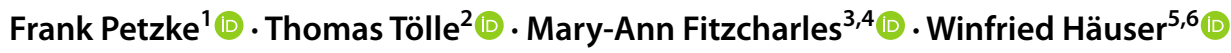

Accepted: 4 November 2021 / Published online: 21 November 2021

(c) The Author(s) 2021

\begin{abstract}
Neuropathic pain represents a broad category of pain syndromes that include a wide variety of peripheral and central disorders. The overall prevalence of neuropathic pain in the general population is reported to be between 7 and $10 \%$. Management of neuropathic pain presents an unmet clinical need, with less than $50 \%$ of patients achieving substantial pain relief with medications currently recommended such as pregabalin, gabapentin, duloxetine and various tricyclic antidepressants. It has been suggested that cannabis-based medicines (CbMs) and medical cannabis (MC) may be a treatment option for those with chronic neuropathic pain. $\mathrm{CbMs} / \mathrm{MC}$ are available in different forms: licensed medications or medical products (plant-derived and/or synthetic products such as tetrahydrocannabinol or cannabidiol); magistral preparations of cannabis plant derivatives with defined molecular content such as dronabinol (tetrahydrocannabinol); and herbal cannabis with a defined content of tetrahydrocannabinol and/or cannabidiol, together with other active ingredients (phytocannabinoids other than cannabidiol/ tetrahydrocannabinol, terpenes and flavonoids). The availability of different types of $\mathrm{CbMs} / \mathrm{MC}$ varies between countries worldwide. Systematic reviews of available randomised controlled trials have stated low-quality evidence for CbMs and MC for chronic neuropathic pain. Depending on the studies included in the various quantitative syntheses, authors have reached divergent conclusions on the efficacy of $\mathrm{CbMs} / \mathrm{MC}$ for chronic neuropathic pain (from not effective to a clinically meaningful benefit). Clinically relevant side effects of $\mathrm{CbMs} / \mathrm{MC}$, especially for central nervous system and psychiatric disorders, have been reported by some systematic reviews. Recommendations for the use of $\mathrm{CbMs} / \mathrm{MC}$ for chronic neuropathic pain by various medical associations also differ, from negative recommendations, no recommendation possible, recommended as third-line therapy, or recommended as an alternative in selected cases failing standard therapies within a multimodal concept. After reading this paper, readers are invited to formulate their own conclusions regarding the potential benefits and harms of $\mathrm{CbMs} / \mathrm{MC}$ for the treatment of chronic neuropathic pain.
\end{abstract}

Winfried Häuser

whaeuser@klinikum-saarbruecken.de

Universitätsmedizin Göttingen, Göttingen, Germany

2 Department of Neurology, Technische Universität München, Munich, Germany

3 Alan Edwards Pain Management Unit, McGill University Health Center, Montreal, QC, Canada

4 Division of Rheumatology, McGill University Health Centre, Quebec, QC, Canada

5 Internal Medicine 1, Klinikum Saarbrücken gGmbH, Winterberg 1, 66119 Saarbrücken, Germany

6 Department of Psychosomatic Medicine and Psychotherapy, Technische Universität München, Munich, Germany

\section{Key Points}

Current systematic reviews on cannabis-based medicines and medical cannabis for chronic neuropathic pain come to divergent conclusions on efficacy.

Recommendations in position papers of international scientific associations and national guidelines differ (from "non-recommended" to "third-line treatment option") for cannabis-based medicines and medical cannabis in the management of chronic neuropathic pain.

Physicians who decide to use cannabis-based medicines or medical cannabis must be mindful of the limited sound evidence for effect and concerns for harms. 


\section{Introduction}

The International Association for the Study of Pain (IASP) has defined neuropathic pain as "pain caused by a lesion or disease of the somatosensory system" [1]. This pain occurs in the absence of a noxious stimulus and may be spontaneous (continuous or paroxysmal) in its temporal characteristics or be evoked by mechanical or thermal stimuli (hyperalgesia/allodynia). Clinically, neuropathic pain syndromes are characterised by a combination of positive and negative phenomena $[2,3]$. The positive phenomena can be identified as various painful symptoms (including spontaneous pain, evoked allodynia, and hyperalgesia to various sensory stimuli), and paraesthesia and/or dysaesthesia, which, by definition, are abnormal non-painful sensations (e.g., tingling, numbness, pins and needles). Negative phenomena usually include neurological sensory deficits in the painful area, together with other deficits (motor or vegetative impairments), depending on the location of the lesion $[2,3]$.

Neuropathic pain represents a broad category of pain syndromes including a wide variety of peripheral or central disorders and is traditionally categorised by the aetiology of diseases affecting the peripheral or central nervous system. Classical aetiologies of peripheral neuropathic pain include painful peripheral neuropathies caused by metabolic (diabetes mellitus or alcohol), inflammatory (post-herpetic neuralgia) or traumatic nerve injury. Central neuropathic pain can be observed in up to $8 \%$ of patients after a stroke, in approximately $30-50 \%$ of patients with a spinal cord injury, a large majority of whom present with a syringomyelia, and up to $20-25 \%$ of patients with multiple sclerosis (MS) [3]. Even more frequent are the mixed pain syndromes involving both neuropathic and non-neuropathic mechanisms, such as lumbar or cervical radiculopathies [4]. In systematic reviews, the overall prevalence of neuropathic pain in the general population is reported to be between 7 and 10\% [5] accounting for $20-25 \%$ of individuals with chronic pain [3].

Neuropathic pain can substantially impair health-related quality of life as it often associates with other problems, such as loss of function, anxiety, depression, disturbed sleep and impaired cognition [6]. The management of neuropathic pain is mostly focused towards treating the symptom, as the underlying cause may be less amenable to treatment. In addition, even when the primary aetiology can be effectively managed, such as with optimal diabetic control, the neuropathic pain usually persists. As neuropathic pain seldom responds to traditional analgesics such as acetaminophen or non-steroidal anti-inflammatory drugs [6], different classes of drugs have been proposed as a therapeutic option. Classes of medications that are recommended include the anti-epileptic drugs (pregabalin, gabapentin), serotonin norepinephrine reuptake inhibitors (duloxetine) and various tricyclic antidepressants. High-concentration capsaicin (the active component of chili peppers) patches, lidocaine (local anaesthetic-blocking sodium channels) patches and tramadol (an opioid receptor agonist with inhibitory effects on serotonin and noradrenaline reuptake) are recommended as second-line treatments for peripheral neuropathic pain, but not central neuropathic pain. Strong opioids and botulinum toxin are recommended for use as third-line treatments [6].

Effective management of neuropathic pain remains an unmet clinical need, with less than $50 \%$ of patients achieving substantial pain relief with medications currently recommended. In addition, adverse effects associated with medications limit their clinical utility [7]. It is plausible that this poor therapeutic outcome may be partially related to failure to impact some relevant pathophysiological mechanisms [3]. Therefore, there is a need to explore other treatment options with different modes of action and from different pharmacological classes.

Cannabis has been promoted by some patient organisations and advocates for the treatment of chronic pain refractory to conventional treatment. Propelled by public advocacy, and contrary to the usual path of drug approval, cannabis flowers and cannabis-based medicines (CbMs) have bypassed traditional evidence-based studies and have been legalised as a therapeutic product by legislative bodies in an increasing number of countries [8]. However, the use of cannabis for medical reasons is highly contested. There remains a lack of robust evidence for efficacy and safety [9] and there are concerns about long-term adverse health effects, with risks extrapolated from those using cannabis for recreational purposes [10]. In addition, systematic reviews on the efficacy and safety of "cannabis" have come to divergent conclusions [11]. We hope that this review will be helpful to a wide audience of physicians who will be managing patients with neuropathic pain including primary care physicians and those of various subspecialists such as neurologists, endocrinologists and rheumatologists, amongst others.

The aims of this review are:

- to explain the rationale why cannabis may hold potential for treatment of chronic neuropathic pain;

- to present an overview of existing systematic reviews on the efficacy and safety of CbMs for chronic neuropathic pain;

- to understand the reasons for different conclusions of the systematic reviews on the efficacy and safety of CbMs for chronic neuropathic pain;

- to summarise the current evidence-based guidelines and position papers on the role of CbMs and medical cannabis (MC) for chronic neuropathic pain; 
- to open debate on some key uncertainties regarding use of CbMs and MC for chronic pain; and

- to provide some guidance for the use of CbMs and MC in clinical practice.

\section{Methods}

We performed a selective search in PubMed from January 2014 to June 2021 with the search terms ("Systematic review" AND "neuropathic pain" AND "cannabis") and with the search terms ("Guideline" and "Neuropathic pain") from 2014 to 2021 . We scanned the reference lists of relevant published articles not identified in the database searches.

\section{Rationale to Use Cannabis for the Management of Chronic Neuropathic Pain}

The endocannabinoid system is ubiquitous in the animal kingdom, with multiple functions that move the organism back to equilibrium. Cannabinoid receptors and ligands can be found in the peripheral and central nervous system, but also in other tissues such as bone and in the immune system. The endocannabinoid neuromodulatory system is involved in multiple physiological functions, such as anti-nociception, cognition and memory, endocrine function, gastrointestinal function, inflammation and immune recognition [12].

The herbal product derived from the plant Cannabis sativa contains over 1000 compounds, with at least 100 classified as phytocannabinoids. Two are of particular medical interest and have been best studied. Delta 9-tetrahydrocannabinol is the main active constituent, with psychoactive (e.g. reduction of anxiety and stress, and the feeling of "high") and pain-relieving properties. The second molecule of interest is cannabidiol (CBD), which has low affinity for the cannabinoid receptors, and with potential to counteract the negative effects of THC on memory, mood and cognition. In addition, CBD may have an independent effect on pain modulation via anti-inflammatory properties [12]. Less studied are the many other molecules, both phytocannabinoid and non-phytocannabinoid, which have been suggested to play a therapeutic role, coined "the entourage effect". The specific roles of currently identified $\mathrm{CbMs}$ that act as ligands at $\mathrm{CB}$ receptors within the nervous system (primarily but not exclusively CB 1 receptors) and in the periphery (primarily but not exclusively CB2 receptors) are only partially elucidated, but with considerable pre-clinical data to support their influence on nociception [12]. It is also hypothesised that cannabis reduces alterations in cognitive, emotional and autonomic processing in chronic pain states [13].

\section{Terminology and Cannabis-Derived Medications Available}

Terminology and definitions of cannabis-derived medicines vary in the literature. In this paper, we use the terminology based on the proposals of the Task Forces of the European Pain Federation (EFIC) [14] and the IASP [15].

\subsection{Licensed Medical Products Approved for Medical Use in Some Countries}

\subsubsection{Plant-Derived Cannabinoids}

These include oromucosal tetrahydrocannabinol (THC) and CBD (nabiximols; Sativex ${ }^{\circledR}$ ) or oral CBD (Epidiolex ${ }^{\circledR}$ ). Nabiximols is approved in some countries for the treatment of refractory spasticity in patients with MS [16]. Oral CBD is approved by the European Medicines Agency for the management of Dravet-Syndrome and Lennaux-Gastaut Syndrome, two rare forms of epilepsy in children [17].

\subsubsection{Synthetic Cannabinoids}

Nabilone $\left(\right.$ Cesamet $^{\circledR}$ or Canemes ${ }^{\circledR}$ ), a synthetic analogue of THC, is approved in some countries for the management of refractory nausea/emesis in patients with cancer [18]. Dronabinol (Marinol ${ }^{\circledR}$ or Syndros ${ }^{\circledR}$ ), as synthetic THC, is approved for similar therapeutic use in some countries [16]. Levonantradol, a potent synthetic THC is currently only available for research, but not as a licensed therapeutic drug in any country.

\subsection{Magistral (Compounded) Preparations of Cannabis Plant Derivatives}

These are defined cannabinoids such as plant-dervied THC $\left(\right.$ Dronabinol ${ }^{\circledR}$ ) and herbal cannabis, resins and extracts, such as oil or tinctures with defined content of THC and/or CBD, together with other active ingredients (phytocannabinoids other than $\mathrm{CBD} / \mathrm{THC}$, terpenes and flavonoids). The leaves and flowers of the plant have the highest concentration of THC and CBD, with a concentration of THC varying from 3 to $30 \%$, and CBD from $<1$ to $13 \%$. Extracts with various THC/CBD combinations range from THC $<1$ to $100 \%$ and CBD $<1$ to $100 \%$. There is a prevalent opinion with only anecdotal evidence that other molecules in the plant such as terpenes and phenolic compounds may synergistically provide a therapeutic effect named "the entourage effect" [18].

The term "dronabinol" is used in different ways: as a generic name for a synthetic THC as a licensed medical product (Marinol ${ }^{\circledR}$ or Syndros ${ }^{\circledR}$ ) in USA and as a generic 
and brand name for a magistral preparation of plant-derived THC in some European countries. The main forms of administration are: (a) oromucosal: spray (nabiximols); (b) oral: capsules (dronabinol, nabilone), oil (CBD) and extracts (dronabinol, herbal cannabis); (c) smoke or vapour inhalation: CBD, plant-derived dronabinol, herbal cannabis and resins; and (d) topical or rectal: CBD, herbal cannabis, resins and extracts.

\subsection{Experimental Medications}

Cannabinoid receptor antagonists and negative allosteric modulators (e.g. rimonabant [SR141716A]), modulators that increase or enhance endocannabinoid system activity (e.g. fatty acid amide hydrolase inhibitors) and synthetic analogues of THC-11-oic acid such as ajulemic acid (AJA, CT-3, IP-751, JBT-101, anabasum) are experimental medications that have been not yet been approved for use in pain therapy outside of clinical studies.

\subsection{Nutritional Supplements}

Cannabidiol and extracts of cannabis flowers (all with THC content of $<0.2 \%,{ }^{<} 0.3 \%$ or $<1.0 \%$ depending on country) are available in many countries as a nutritional supplement [19].

\section{Systematic Reviews with a Meta-Analysis of Randomized Controlled Trials (RCTs) with CbMs for Chronic Neuropathic Pain}

Reviews differed markedly with regard to databases searched, the inclusion criteria, study duration, the types of $\mathrm{CbMs} / \mathrm{MC}$ tested and the outcomes analysed (see Table 1). The search for systematic reviews with a metaanalysis yielded 16 hits. One systematic review [20] presented a subgroup analysis of six studies with THC/CBD spray in chronic neuropathic pain with an odds ratio for pain relief of $30 \%$ and greater of 1.38 (95\% confidence interval 0.93-2.03). This subgroup analysis for chronic neuropathic pain did not include other cannabinoids that were included in the qualitative analysis. Therefore, this systematic review was not included in Table 1.

\subsection{Study Duration and Pain Condition}

The most recent literature search (until June 2020) was conducted by Dykukha et al. [21], and the most extensive search was for the Cochrane review of Mücke et al. [22]. Mücke et al. [22] and Petzke et al. [23] required at least 2 weeks of treatment to include studies for quantitative analysis. Finnerup et al. required at least a 3-week double-blind duration of the RCTs for inclusion [7]. In contrast, other reviews included RCTs with a $\leq 1$ day duration (experimental studies) [24, 25]. The most frequently analysed pain syndromes were peripheral neuropathies of different origins (e.g. chemotherapy, diabetes, human immunodeficiency virus), traumatic lesions (peripheral nerve, plexus, spinal cord), and MS-associated spinal and cerebral neuropathic pain.

\subsection{Cannabis-Derived Medications Studied}

Andreae et al. analysed only RCTs with inhaled MC [24] and Dykukha et al. only studies with nabiximols [21]. Finnerup et al. [7], Mücke et al. [22] and Petzke et al. [23] analysed only RCTs with CbMs (dronabinol, nabilone, nabiximols) and MC available for clinical use. Only Fisher et al. included RCTs with experimental medications (e.g. fatty acid amide hydrolase inhibitors) [25] (see Table 1). Therefore, the intersection of RCTs included in the systematic review is only large between the Mücke et al. [22] and Petzke et al. [23] studies (Table 1 of the Electronic Supplementary Material).

\subsection{Outcomes for Treatments with Cannabis-Derived Medications}

The $95 \%$ confidence interval for the outcome of 30\% pain relief or more included zero in the meta-analysis of Finnerup et al. [7] and in the analysis of studies of $>4$ weeks' duration by Fisher et al. [25]. The meta-analyses of the other authors found statistically significant effects of CbMs and MC with the number needed to benefit between 6 and 14 for pain relief of 30\% or more [21-23]. Andreae et al. [24], Dykukha et al. [21] and Fisher et al. [25] did not analyse adverse outcomes. The number needed to harm for drop-outs due to adverse events ranged between 12 and 25 in the other reviews [22, 23]. The number needed to harm for psychiatric disorders side effects ranged between 8 and 10 [22, 23]. Serious adverse events did not differ between $\mathrm{CbMs} / \mathrm{MC}$ and placebo in two meta-analyses [22, 23]. A rating of the methodological quality of the RCTs analysed according to GRADE was only performed by Fisher et al. [25] and Mücke et al. [22]. Both reviews stated a very low to low quality evidence for the RCTs analysed.

\subsection{Conclusions Based on Current Systematic Reviews and Meta-Analyses}

The conclusions of the authors for clinical practice are summarised in Table 2. Finnerup et al. gave a weak recommendation against the use of $\mathrm{CbMs} / \mathrm{MC}$ [7]. Petzke et al. concluded that $\mathrm{CbMs}$ can be considered as third-line therapies [23]. Four reviews gave no recommendations for clinical practice. Andreae et al. [24] and Dykukha et al. [21] 


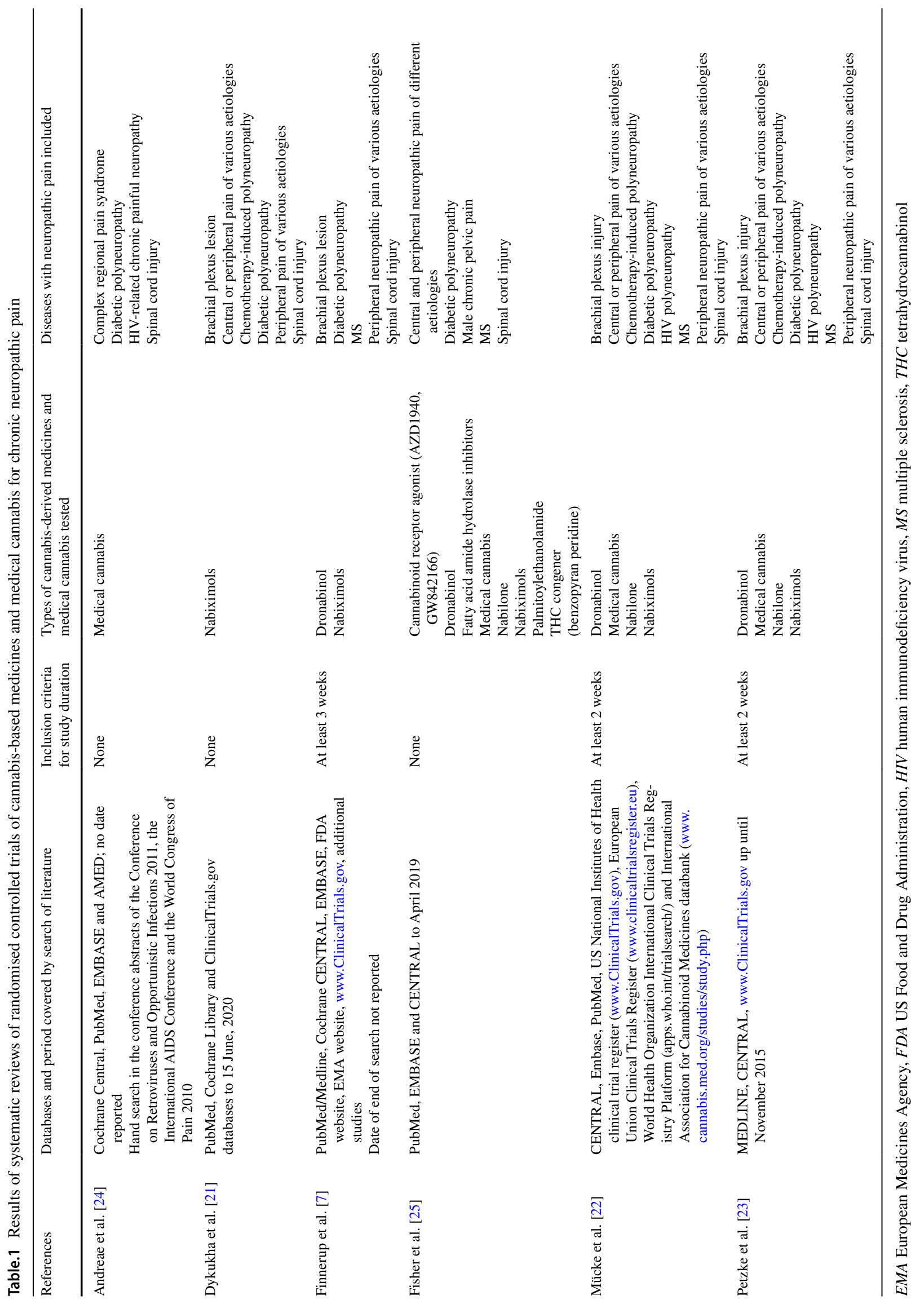




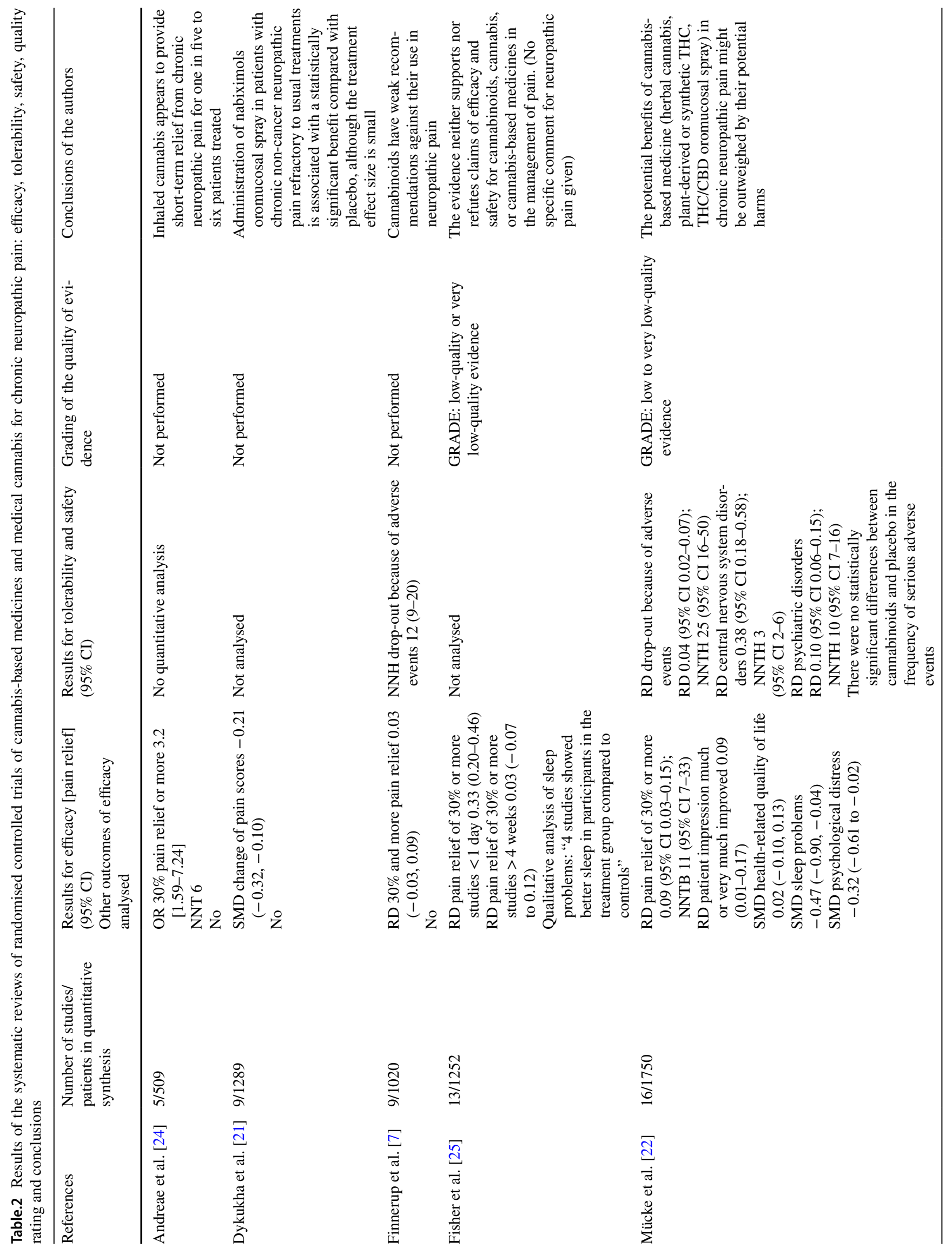




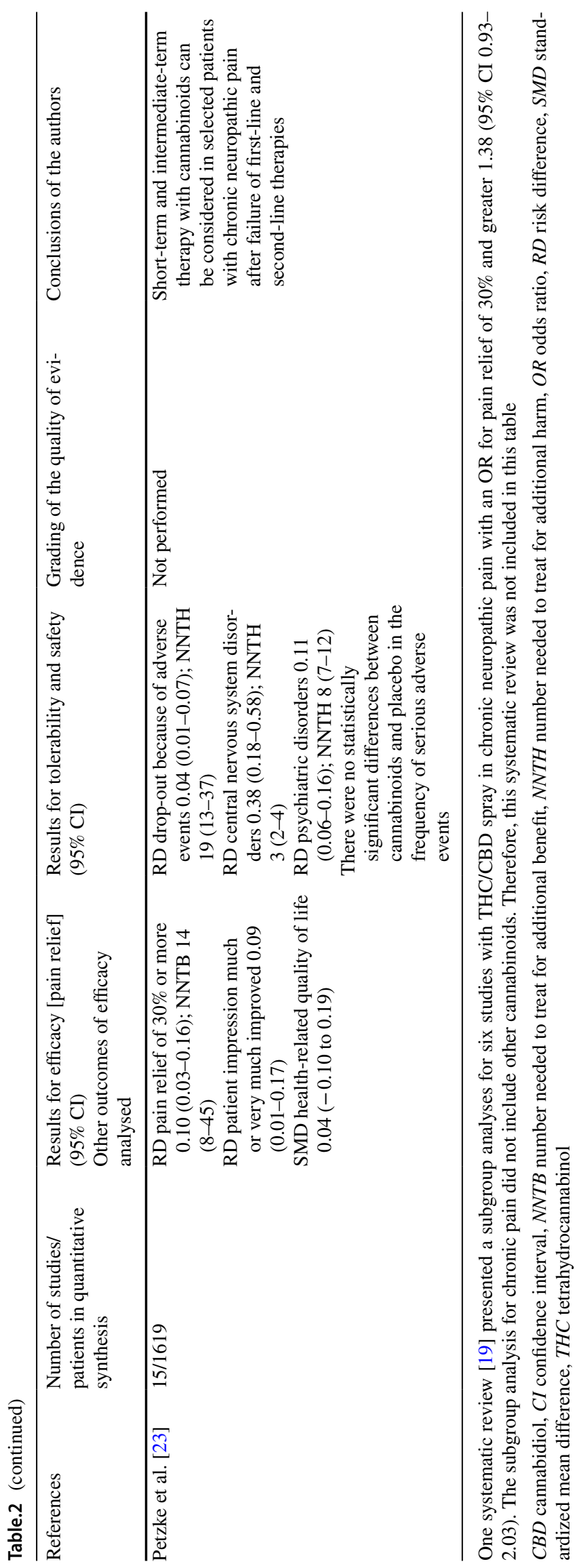


summarised studies of MC and nabiximols, respectively, with both concluding that there was short-term relief of neuropathic pain. Fisher et al. concluded that the evidence neither supports nor refutes claims of efficacy and safety of CbMs [25]. Mücke et al. stated that the potential benefits of CbMs might be outweighed by their potential harms [22].

\section{Long-Term Studies with CbMs for Chronic Neuropathic Pain}

We found no long-term RCT with $\mathrm{CbMs} / \mathrm{MC}$ to address the question of long-term efficacy and safety. We have identified four studies that can be used to help inform clinicians about the long-term effects of CbMs [26-29]. Follow-up time was 32 weeks, 38 weeks, 12 months and 12 weeks. Overall pain reduction and other parameters such as global well-being and sleep improvement were maintained over the long term, side effects were mostly mild, and the study from Israel reported a reduction in opioid dose.

One RCT with dronabinol for MS-associated neuropathic pain was extended to a 32-week open-label period. During this long-term follow-up, pain intensities remained at a low level (range $2.5-3.8$ of a $0-10$ scale), with a number of adverse events and dropouts due to adverse events lower in the long term than in the randomised controlled period. "Mild signs" of drug dependency were documented for one participant [26].

In a 38-week open-label extension study of two RCTs, THC/CBD oromucosal spray was added to current analgesic treatment for 380 participants with diabetes-associated polyneuropathy or allodynia. The proportion of participants who reported at least a clinically relevant $30 \%$ improvement in pain continued to increase with time (up to 9 months); at least half of all participants reported a $30 \%$ improvement at all timepoints. Improvements were observed for all secondary efficacy outcomes, including sleep quality, Patient Global Impression of Change and health-related quality of life. The THC/CBD spray was well tolerated for the study duration, participants did not seek to increase their dose with time and there were no new safety concerns arising from long-term use [27].

A study of patients with all types of chronic pain licensed to use MC in Israel included 1045 patients at baseline, with 551 completing the 12-month follow-up. At 1 year, average pain intensity declined from baseline by $20 \%$ [ -1.97 points (95\% confidence interval -2.13 to -1.81$)$ ], and all other parameters improved by $10-30 \%(p<0.001)$. The morphine equivalent daily dosage of opioids was significantly decreased by $42 \%$ (reduction of $27 \mathrm{mg}$; $95 \%$ confidence interval -34.89 to -18.56 ) from baseline. Reported adverse events were common but mostly non-serious. Relatively higher treatment success was predicted by the presence of a normal to long sleep duration, lower body mass index and a lower depression score, whereas the presence of neuropathic pain predicted the opposite [28].

An exploratory analysis of anonymised 12-week routine/ open-label data provided by the German Pain e-Registry on adult patients with severe chronic pain treated with THC:CBD oromucosal spray in 2017 included 800 patients of whom $248(31.0 \%)$ reported conditions and International Classification of Diseases, 10th Revision diagnoses usually categorised as neuropathic. Average daily dose was THC $19.2 \pm 3.8 \mathrm{mg}$ (median: 18.9, range: $8.1-29.7$ ) and CBD $17.8 \pm 3.5 \mathrm{mg}$ (median: 17.5, range: $7.5-27.5$ ) at the 12 -week treatment end. An aggregated nine-factor symptom relief score was $54.9 \pm 17.2$ percent for patients with neuropathic chronic pain, a score significantly higher than that recorded by patients with a mixed-type chronic pain $(18.2 \pm$ $12.0)$ or those with nociceptive chronic pain $(-11.9 \pm 10.5)$. Over three quarters $(76.1 \%)$ of patients with neuropathic pain reported that their overall situation was "much better" or even "very much better" because of THC:CBD treatment. Adverse events reported by $19.1 \%$ of the total sample, were of mild $(81.6 \%)$ or moderate intensity $(16.5 \%)$, and were either self-limiting or easily tolerated over time. Only four events (two for fatigue, one each for dysgeusia and dizziness) were classified as severe [29].

\section{Guidelines and Position Papers on CbMs for Chronic Neuropathic Pain}

The search yielded 262 hits and we identified that seven working groups/societies had published guidance for the use of $\mathrm{CbMs} / \mathrm{MC}$ in the management of chronic pain conditions. Three groups (the Canadian Pain Society, the EFIC, the German Pain Society) recommended CbMs as a thirdline treatment option for chronic neuropathic pain [14, 30, 31]. The German Society of Neurology recommended only off-label use in conjunction with a multimodal treatment strategy [32]. The National Institute for Health Care Excellence (UK), French Society of Neurology and the IASP all recommended against the use of $\mathrm{CbMs} / \mathrm{MC}$, citing the lack of high-quality evidence [33-35]. We are aware that EFIC's recommendation is contrary to the recent National Institute for Health Care Excellence, IASP French Chapter and the IASP positions. We however have taken the stance that CbMs are being used commonly by patients, including those with neuropathic pain, and we believe that the EFIC's recommendation better addresses the realities of clinical practice whereby many clinicians recommend this treatment as third-line and fourth-line therapies.

In 2014, the Canadian Pain Society recommended cannabinoids as a third-line treatment option [30]. In 2018, the Task Force of the European Pain Federation stated 
that $\mathrm{CbMs} / \mathrm{MC}$ can be considered as third-line therapy for chronic neuropathic pain [14]. This recommendation was adopted by the German Pain Society in the position paper of 2019 [31].

In 2019, the German Society of Neurology published a new guideline on the diagnosis and non-interventional therapy for any aetiology neuropathic pain, but with the exclusion of trigeminal neuralgia and complex regional pain syndrome. Cannabinoids were not recommended and could only be considered as off-label therapy within a multimodal therapy concept [32].

In 2020, the National Institute for Health and Care Excellence (UK) recommended that treatment should not be initiated with the cannabis sativa extract for neuropathic pain [33]. In 2020, the IASP French Chapter and the French Society of Neurology concluded that there was inconclusive evidence for the use of cannabinoids (oromucosal nabiximols, oral THC) in neuropathic pain because of the large number of high-quality trials yielding negative results [34].

In March 2021, the IASP issued a statement that use of cannabinoids for the treatment of pain cannot, at this time, be endorsed because of a lack of evidence from high-quality research. A qualifier to this statement is that the lived experiences of people with pain who have found benefit from use of cannabinoids cannot be dismissed [35].

\section{Some Open Questions}

\subsection{Distinct Neuropathic Pain Syndromes}

Although the RCTs included in the systematic reviews included the most common neuropathic pain syndromes such as peripheral polyneuropathy of different origins and MS-associated neuropathic pain, several neuropathic pain syndromes such as trigeminal neuralgia and post-stroke central pain were either not or rarely included in the studies. Therefore, there is a lack of evidence for CbMs in these disease entities.

\subsection{Neuropathic Pain Profiles}

Persons with chronic neuropathic pain exhibit variable painrelated symptoms and signs [2, 3], and use different pain descriptors (e.g. burning, tugging, pricking, cramping), leading pain experts to advocate for a more tailored approach. It is suggested that clinical profiles of symptoms and signs may indeed reflect different pathophysiological mechanisms $[36,37]$. Furthermore, the value of a standardised bedside examination, quantitative sensory testing and neuropathic pain questionnaires remains a matter of debate [38] Only one study has focussed on a potential mechanism for the effect of cannabinoids on neuropathic pain [39]. The presence of allodynia was associated with a significant difference in the $30 \%$ responder rate and also in some secondary outcomes (sleep quality and global impression of change) for nabiximols compared with placebo. However, neither the neuropathic pain scale score nor dynamic or punctuate allodynia improved significantly [39].

\subsection{Safety}

A major concern often raised by psychiatrists and some politicians is the risk of abuse and dependence when $\mathrm{CbMs} / \mathrm{MC}$ are used to manage chronic pain. These concerns are mostly derived from data on recreational cannabis use, where cannabis use disorder is reported with a prevalence of up to $10 \%$. Indeed, extrapolating this risk to persons using $\mathrm{CbMs} /$ $\mathrm{MC}$ as a therapy is problematic [10].

There are currently only a few studies that address abuse and dependence in patients with chronic pain prescribed $\mathrm{CbMs} / \mathrm{MC}$. A cross-sectional study in two Israeli pain centres found the prevalence of problematic use of cannabis according to Diagnostic and Statistical Manual of Mental Disorders (DSM), 4th Edition to be 21.2\% [40]. However, the use of DSM, 4th Edition and International Classification of Diseases, 10th Revision criteria for dependence and of DSM, 5th Edition for use disorder in patients with prescription $\mathrm{CbMs} / \mathrm{MC}$ remains ambiguous [41]. From the perspective of managing patients with chronic pain, some behaviours that are considered symptoms of dependence/ use disorder in the International Classification of Diseases, 10th Revision and DSM, 4th Edition and DSM, 5th Edition might result from insufficient pain control or reflect tolerance due to long-term use [41, 42]. In Germany, physicians who prescribe MC flowers or CbMs (requiring a narcotic prescription) reimbursed by the statutory health insurance are required to participate in an accompanying non-interventional survey that runs until 31 March, 2022. At the last interim evaluation on 11 May, 2020, there were 10,010 completed datasets, still only reflecting a subset of patients actually treated, as participation in the survey is not controlled or obligatory. Pain was the most frequently treated symptom in $73 \%$. Abuse and dependence (according to the clinical impression of the prescribing physician) were reported for $0.1 \%$ of the patients in the whole sample [43]. The position papers of the EFIC [13] and the German Pain Society [31] state that the risk of abuse (e.g. diversion, use for illicit purposes) is probably higher for inhaled cannabis strains with high THC content than for oral cannabis-based medicines or inhaled cannabis strains with a low THC content [31].

Another concern is the negative effects on the driving ability of cannabis consumers. In contrast to recreational use of mostly inhaled cannabis products, which has been associated with increased motor vehicle accidents, little is known 
about medical use and driving safety [44] but remains a relevant concern for all cannabis-containing products. Finally, drug interactions must be considered [44].

Mohiuddin et al. concluded in a recent systematic review on general risks of harm that cannabis exposure is associated with higher risks of psychosis, motor vehicle accidents, respiratory problems, testicular cancer, low birth weight and short-term adverse events. However, these findings are mainly derived from settings of non-medicinal use [44].

\subsection{Reliability of the Systematic Reviews on CbMs for Chronic Pain}

A systematic overview of systematic reviews of CbMs for pain management stated that the quality of most systematic reviews on cannabinoids for pain was critically low or low except for the quality of the Cochrane review on cannabinoids for chronic neuropathic pain [22], which was graded as high [45].

\section{Discussion}

Any discussion of the potential clinical use of $\mathrm{CbMs} / \mathrm{MC}$ for chronic neuropathic pain must acknowledge the large differences between countries regarding availability, approval and reimbursement of $\mathrm{CbMs} / \mathrm{MC}$ [16]. In addition, the personal attitudes and preferences of physicians may differ between countries. In Canada, Israel and the USA, cannabis flowers are the most commonly prescribed cannabis preparation for chronic pain [9], whereas German physicians prefer to prescribe oral dronabinol (plant-derived THC) [43]. The current evidence does not favour any single $\mathrm{CbM}$ or $\mathrm{MC}$ preparation [22]. Furthermore, the evidence available is biased for many reasons: most of the RCTs were conducted with nabiximols and sponsored by the manufacturer of the drug and performed mostly in cannabis-naive patients with chronic pain; most patients in the other MC studies were past or current recreational cannabis users [22]; and the studies have been sponsored by public grants or institutions.

The reader of systematic reviews and guidelines on $\mathrm{CbMs} / \mathrm{MC}$ for chronic neuropathic pain is confronted with divergent results, conclusions and recommendations. The authors of this paper have contributed to some systematic reviews and position papers [14, 22, 23, 39, 46, 47], and therefore could be biased in their position towards the use of $\mathrm{CbMs} / \mathrm{MC}$ for chronic neuropathic pain.

The results of the systematic reviews on efficacy range from "effective" to "not effective". The discrepancy can be partially explained by inclusion criteria, study duration and the number of studies included in individual quantitative analyses. For example, if Finnerup et al. [7] had included studies of 2 weeks' duration, they would have found statistical superiority of $\mathrm{CbMs} / \mathrm{MC}$ over placebo for pain relief [11]. Other potential positive effects of $\mathrm{CbMs} / \mathrm{MC}$ for patients with chronic neuropathic pain were studied by three of the systematic reviews assessed. Reducing sleep problems and psychological distress in patients with chronic neuropathic pain as demonstrated by the systematic review of Mücke et al. [22] should be included in the clinical decision making with the patient. When reporting on the tolerability of $\mathrm{CbMs} / \mathrm{MC}$, drop-out rates because of adverse events were comparable to those seen for other centrally acting medications such as anticonvulsants, antidepressants and opioids as described in the review of Finnerup et al. [7]. A finding of less than ten for the number needed to harm for adverse events with central nervous system disorders and psychiatric disorders reported in two reviews indicates a clinically relevant potential harm [22,23]. Severe adverse events related to treatment with $\mathrm{CbM} / \mathrm{MC}$ are very rare, but with a cautionary note that the same holds true for opioid studies in a well-controlled environment and may not capture the risks of broader use [46]. These considerations should be part of the shared decision making with patients who may perceive that $\mathrm{CbMs} / \mathrm{MC}$ are less harmful because they are "natural".

\section{Recommendations for Clinical Practice}

Those readers who decide to use $\mathrm{CbMs} / \mathrm{MC}$ as a third-line option/individual therapeutic trial for patients with chronic neuropathic pain might consider these recommendations of recent guidelines and position papers. The general principles of treatment with centrally acting analgesics such as opioids [47] are also valid for CbMs/MC (see Table 3).

Pragmatic guidance for prescription use of $\mathrm{CbMs} / \mathrm{MC}$ has been provided by the Canadian Association of Rheumatology [46]. Ideally, a licensed pharmaceutical product or a magistral preparation should be tried prior to herbal products, although this may not be possible in many jurisdictions, and for some, cost may be prohibitive. The content of ingested THC and/or CBD is better defined in these products than in MC. The bioavailability of inhaled cannabis is more variable than of oral (pills, oils) CbMs. Innovative devices might reduce the concerns about inhalation of cannabis flowers. In an experimental RCT with patients with chronic neuropathic pain, a novel selective-dose cannabis inhaler delivered significantly lower and more precise doses of THC, thus allowing the administration of inhaled CbMs according to high pharmaceutical standards [48].

Any treatment with cannabis products should be prescribed and managed by a physician fully knowledgeable and responsible for the patient care. Management should not be in the hands of non-medical "cannabis experts". If a herbal cannabis product is prescribed, an oil preparation for oral consumption with mostly CBD and low THC (to 
Table.3 Algorithmic approach for cannabis-based medicines for chronic neuropathic pain (modified according to [46])

1. Comprehensive clinical evaluation

(a) Medical and psychosocial history

(b) Medical and if necessary psychological and physical examination

(c) Technical examinations

(d) Interdisciplinary assessment if needed

2. Start multimodal treatment

(a) Education

(b) Non-pharmacological therapies

(c) Start with first-line and second-line therapies (e.g. duloxetine, gabapentin, pregabalin) according to patients' comorbidities

3. Consider a trial with cannabis-based medicines or medical cannabis if

(a) Established pharmacological and non-pharmacological therapies are

(i) Not effective and /or

(ii) Not tolerated and/or

(iii) Contraindicated

4. Shared decision making with patients when considering a trial of cannabis-based medicines or medical cannabis

(a) Assess individual benefit-risk ratio

(b) Obtain informed consent and agreement (depending on jurisdiction and regulations)

(c) Establish individual and realistic treatment goals (sustained improvement of daily functioning, pain reduction)

5. Initial dose adjustment phase (4 weeks)

(a) Start slow, go slow ${ }^{\text {a }}$

(b) Monitor response and assess for side effects

(c) Find the optimal dosage (predefined treatment goals met; tolerable/manageable side effects)

(d) Discontinue if

(i) Predefined treatment goals not reached

(ii) Intolerable/unmanageble side effects

(iii) Abuse/misuse of prescribed cannabis-based medicines/medical cannabis

6. Long-term therapy (>12 weeks)

(a) Regular assessments (at least every 3 months)

(b) Assess four As: activity, analgesia, aberrant behaviour, adverse effects

(c) Promote non-pharmacological therapies

(d) Continue if

(i) Stable dosage

(ii) Sustained improvement of daily functioning and pain reduction

(iii) Tolerable/manageabale side effects

(iv) No signals of abuse/misuse of prescribed cannabis-based medicines/medical cannabis

(e) Discuss tapering /drug holiday after 6 months with the patient

(f) Discontinue if

(i) Dose escalation

(ii) Loss of improvement of daily functioning and of pain reduction

(iii) Tolerable/manageable side effects

(iv) Signals of abuse/misuse of prescribed cannabis-based medicines/medical cannabis

CBD cannabidiol, $T H C$ tetrahydrocannabinol

${ }^{a}$ Recommended starting dosage for nabiximols: 2 puffs (5.4 mg THC and $5 \mathrm{mg}$ CBD); increase of 1 or 2 puffs every second day; maximum dosage: 12 puffs (32.4 mg THC and $30 \mathrm{mg} \mathrm{CBD)/day,} \mathrm{Recommended} \mathrm{starting} \mathrm{dosage} \mathrm{for} \mathrm{nabilone:} \mathrm{starting} \mathrm{dosage} 2.5 \mathrm{mg}$ (split three times/24 hours); increase by $0.8 \mathrm{mg}$ every second day; dose range $5-30 \mathrm{mg}$ /day 
minimise psychoactive effects) is recommended and should be obtained from a regulated licensed medical grower/facility [45].

Patients should be discouraged from smoking or vaping of cannabis flowers, as dosing is poorly defined. Patients can be directed to authentic sites to obtain up-to-date and valid medical information, e.g. Dutch Office of Medicinal Cannabis 2019 [49].

\section{Conclusions}

At this time, there remains much uncertainty as to the true place for $\mathrm{CbMs} / \mathrm{MC}$ as a therapeutic option for patients with chronic neuropathic pain. Public advocacy, anecdotal reports and poor-quality scientific comment cannot override sound evidence. More rigorous and robust research is needed to better understand the potential benefits and harms of CbMs/ MC for pain relief, and to ensure the safety of patients and the public through regulatory standards and safeguards [35]. More real-world data with standardised and appropriate instruments are needed to assess whether abuse/dependence/ cannabis use disorder from prescribed $\mathrm{CbMs} / \mathrm{MC}$ may be a clinically relevant problem, and to identify populations at risk and institute preventive measures.

Supplementary Information The online version contains supplementary material available at https://doi.org/10.1007/s40263-021-00879-w.

\section{Declarations}

Funding Open Access funding enabled and organized by Projekt DEAL

Conflict of interest WH, FP and MAF have no financial conflicts of interest to declare. WH is the head of EFIC's Task Force of a position paper on $\mathrm{CbMs}$ and $\mathrm{MC}$ for chronic pain and a member of the Task Force of the German Pain Society on the same topic. FP is the head of the Task Force of the German Pain Society of a position paper on $\mathrm{CbMs}$ and $\mathrm{MC}$ for chronic pain. MAF is the head of a Task Force of the Canadian Association of Rheumatology of a position paper on MC for rheumatic diseases. TT has received honoraria for consultancies, travel grants and speaking fees for AOP Orphan, Almiral Hermal, Bionest Partners, Benkitt Renkiser, Grünenthal, Hexal, Indivior, Kaia Health, Lilly, Medscape, Mundipharma, MSD, Novartis, Pfizer, Recordati Pharma, Sanofi-Aventis and TAD Pharma.

Ethics approval Not applicable.

Consent to participate Not applicable.

Consent for publication Not applicable.

Availability of data and material Not applicable.

Code availability Not applicable.
Author contributions All authors participated in writing the manuscript.

Open Access This article is licensed under a Creative Commons Attribution-NonCommercial 4.0 International License, which permits any non-commercial use, sharing, adaptation, distribution and reproduction in any medium or format, as long as you give appropriate credit to the original author(s) and the source, provide a link to the Creative Commons licence, and indicate if changes were made. The images or other third party material in this article are included in the article's Creative Commons licence, unless indicated otherwise in a credit line to the material. If material is not included in the article's Creative Commons licence and your intended use is not permitted by statutory regulation or exceeds the permitted use, you will need to obtain permission directly from the copyright holder. To view a copy of this licence, visit http://creativecommons.org/licenses/by-nc/4.0/.

\section{References}

1. Jensen TS, Baron R, Haanpää M, Kalso E, Loeser JD, Rice AS. A new definition of neuropathic pain. Pain. 2011;152:2204-5. https://doi.org/10.1016/j.pain.2011.06.017.

2. Maier C, Baron R, Tölle TR, Binder A, Birbaumer N, Birklein F, Gierthmühlen J, et al. Quantitative sensory testing in the German Research Network on Neuropathic Pain (DFNS): somatosensory abnormalities in 1236 patients with different neuropathic pain syndromes. Pain. 2010;150:439-50. https://doi.org/ 10.1016/j.pain.2010.05.002.

3. Bouhassira D. Neuropathic pain: definition, assessment and epidemiology. Rev Neurol (Paris). 2019;175:16-25. https://doi.org/ 10.1016/j.neurol.2018.09.016.

4. Bouhassira D, Lanteri-Minet M, Attal N, Laurent B, Touboul C. Prevalence of chronic pain with neuropathic characteristics in the general population. Pain. 2008;136:380-7. https://doi.org/ 10.1016/j.pain.2007.08.013.

5. Van Hecke O, Austin SK, Khan RA, Smith BH, Torrance N. Neuropathic pain in the general population: a systematic review of epidemiological studies. Pain. 2014;155:654-62. https://doi. org/10.1016/j.pain.2013.11.013.

6. Colloca L, Ludman T, Bouhassira D, Baron R, Dickenson AH, Yarnitsky D, et al. Neuropathic pain. Nat Rev Dis Primers. 2017;3:17002. https://doi.org/10.1038/nrdp.2017.2.

7. Finnerup NB, Attal N, Haroutounian S, McNicol E, Baron R, Dworkin RH, et al. Pharmacotherapy for neuropathic pain in adults: a systematic review and meta-analysis. Lancet Neurol. 2015;14:162-73. https://doi.org/10.1016/S1474-4422(14) 70251-0.

8. Fitzcharles MA, Eisenberg E. Medical cannabis: a forward vision for the clinician. Eur J Pain. 2018;22:485-91. https:// doi.org/10.1002/ejp.1185.

9. Fitzcharles MA, Shir Y, Häuser W. Medical cannabis: strengthening evidence in the face of hype and public pressure. CMAJ. 2019;19:E907-8. https://doi.org/10.1503/cmaj.190509.

10. Volkow ND, Baler RD, Compton WM, Weiss SR. Adverse health effects of marijuana use. N Engl J Med. 2014;370:221927. https://doi.org/10.1056/NEJMra1402309.

11. Häuser W, Petzke F, Fitzcharles MA. Efficacy, tolerability and safety of cannabis-based medicines for chronic pain management: an overview of systematic reviews. Eur J Pain. 2018;22:455-70. https://doi.org/10.1002/ejp.1118.

12. Finn DP, Haroutounian S, Hohmann AG, Krane E, Soliman N, Rice ASC. Cannabinoids, the endocannabinoid system, and 
pain: a review of preclinical studies. Pain. 2021;162(Suppl. 1):S5-25. https://doi.org/10.1097/j.pain.0000000000002268.

13. Guindon J, Hohmann AG. The endocannabinoid system and pain. CNS Neurol Disord Drug Targets. 2009;8:403-29. https:// doi.org/10.2174/187152709789824660.

14. Häuser W, Finn DP, Kalso E, Krcevski-Skvarc N, Kress HG, Morlion B, et al. European Pain Federation (EFIC) position paper on appropriate use of cannabis-based medicines and medical cannabis for chronic pain management. Eur J Pain. 2018;22:1547-64. https://doi.org/10.1002/ejp.1297.

15. Soliman N, Hohmann AG, Haroutounian S, Wever K, Rice ASC, Finn DP. A protocol for the systematic review and meta-analysis of studies in which cannabinoids were tested for antinociceptive effects in animal models of pathological or injury-related persistent pain. Pain Rep. 2019;4: e766. https://doi.org/10.1097/ PR9.0000000000000766.

16. Krcevski-Skvarc N, Wells C, Häuser W. Availability and approval of cannabis-based medicines for chronic pain management and palliative/supportive care in Europe: a survey of the status in the chapters of the European Pain Federation. Eur J Pain. 2018;22:440-54. https://doi.org/10.1002/ejp.1147.

17. European Medicines Agency. Epidyolex. 2019. Available from: https://www.ema.europa.eu/en/medicines/human/EPAR/epidy olex. Accessed 1 June 2020.

18. Andre CM, Hausman JF, Guerriero G. Cannabis sativa: the plant of the thousand and one molecules. Front Plant Sci. 2016;7:19. https://doi.org/10.3389/fpls.2016.00019.

19. Radbruch L, Häuser W. Cannabidiol [in German]. Schmerz. 2020;34:115-6. https://doi.org/10.1007/s00482-020-00458-w.

20. Whiting PF, Wolff RF, Deshpande S, Di Nisio M, Duffy S, Hernandez AV, et al. Cannabinoids for medical use: a systematic review and meta-analysis. JAMA. 2015;313:245673. https://doi.org/10.1001/jama.2015.6358 (Erratum in: JAMA 2015;314:520. Erratum in: JAMA 2015;314:837. Erratum in: JAMA 2015;314:2308. Erratum in: JAMA 2016;315:1522).

21. Dykukha I, Malessa R, Essner U, Überall MA. Nabiximols in chronic neuropathic pain: a meta-analysis of randomized placebocontrolled trials. Pain Med. 2021;22:861-74. https://doi.org/10. 1093/pm/pnab050.

22. Mücke M, Phillips T, Radbruch L, Petzke F, Häuser W. Cannabisbased medicines for chronic neuropathic pain in adults. Cochrane Database Syst Rev. 2018;3:CD012182. https://doi.org/10.1002/ 14651858.CD012182.pub2.

23. Petzke F, Enax-Krumova EK, Häuser W. Efficacy, tolerability and safety of cannabinoids for chronic neuropathic pain: a systematic review of randomized controlled studies [in German]. Schmerz. 2016;30:62-88. https://doi.org/10.1007/s00482-015-0089-y (Erratum in: Schmerz. 2017 Sep 6; PMID: 26830780).

24. Andreae MH, Carter GM, Shaparin N, Suslov K, Ellis RJ, Ware MA, et al. Inhaled cannabis for chronic neuropathic pain: a metaanalysis of individual patient data. J Pain. 2015;16:1221-32. https://doi.org/10.1016/j.jpain.2015.07.009.

25. Fisher E, Moore RA, Fogarty AE, Finn DP, Finnerup NB, Gilron I, et al. Cannabinoids, cannabis, and cannabis-based medicine for pain management: a systematic review of randomised controlled trials. Pain. 2021;162(Suppl. 1):S45-66. https://doi.org/10.1097/j. pain.0000000000001929.

26. Schimrigk S, Marziniak M, Neubauer C, Kugler EM, Werner G, Abramov-Sommariva D. Dronabinol is a safe long-term treatment option for neuropathic pain patients. Eur Neurol. 2017;78:320-9. https://doi.org/10.1159/000481089.

27. Hoggart B, Ratcliffe S, Ehler E, Simpson KH, Hovorka J, Lejčko $\mathrm{J}$, et al. A multicentre, open-label, follow-on study to assess the long-term maintenance of effect, tolerance and safety of THC/ CBD oromucosal spray in the management of neuropathic pain. J Neurol. 2015;262:27-40. https://doi.org/10.1007/ s00415-014-7502-9.

28. Aviram J, Pud D, Gershoni T, Schiff-Keren B, Ogintz M, Vulfsons $\mathrm{S}$, et al. Medical cannabis treatment for chronic pain: outcomes and prediction of response. Eur J Pain. 2021;25:359-74. https:// doi.org/10.1002/ejp.1675.

29. Ueberall MA, Essner U, Mueller-Schwefe GH. Effectiveness and tolerability of THC:CBD oromucosal spray as add-on measure in patients with severe chronic pain: analysis of 12-week open-label real-world data provided by the German Pain e-Registry. J Pain Res. 2019;12:1577-604. https://doi.org/10.2147/JPR.S192174.

30. Moulin D, Boulanger A, Clark AJ, Clarke H, Dao T, Finley GA, Canadian Pain Society, et al. Pharmacological management of chronic neuropathic pain: revised consensus statement from the Canadian Pain Society. Pain Res Manag. 2014;19:328-35. https:// doi.org/10.1155/2014/754693.

31. Petzke F, Karst M, Gastmeier K, Radbruch L, Steffen E, Häuser W, Ad-hoc-Kommission der Deutschen Schmerzgesellschaft "Cannabis in der Medizin." Position paper on medical cannabis and cannabis-based medicines in pain medicine [in German]. Schmerz. 2019;33:449-65. https://doi.org/10.1007/s00482-019-00407-2.

32. Schlereth T. Guideline, "diagnosis and non interventional therapy of neuropathic pain" of the German Society of Neurology (deutsche Gesellschaft für Neurologie). Neurol Res Pract. 2020;2:16. https://doi.org/10.1186/s42466-020-00063-3.

33. National Institute for Health and Care Excellence Neuropathic pain in adults: pharmacological management in non-specialist settings. London: National Institute for Health and Care Excellence (UK). 2020. https://www.ncbi.nlm.nih.gov/books/NBK552848/ pdf/Bookshelf_NBK552848.pdf. Accessed 1 June 2021.

34. Moisset X, Bouhassira D, Avez Couturier J, Alchaar H, Conradi $\mathrm{S}$, Delmotte $\mathrm{MH}$, et al. Pharmacological and non-pharmacological treatments for neuropathic pain: systematic review and French recommendations. Rev Neurol (Paris). 2020;176:325-52. https:// doi.org/10.1016/j.neurol.2020.01.361.

35. Haroutounian S, Arendt-Nielsen L, Belton J, Blyth FM, Degenhardt L, Di Forti M, et al. International Association for the Study of Pain Presidential Task Force on Cannabis and Cannabinoid Analgesia: research agenda on the use of cannabinoids, cannabis, and cannabis-based medicines for pain management. Pain. 2021;162(Suppl. 1):S117-24. https://doi.org/10.1097/j.pain. 0000000000002266.

36. Tölle TR, Baron R, de Bock E, Junor R, Dias Barbosa C, Marshall $\mathrm{SF}$, et al. painPREDICT: first interim data from the development of a new patient-reported pain questionnaire to predict treatment response using sensory symptom profiles. Curr Med Res Opin. 2019;35:1177-85. https://doi.org/10.1080/03007995.2018.15626 87.

37. Bouhassira D, Branders S, Attal N, Fernandes AM, Demolle D, Barbour J, et al. Stratification of patients based on the Neuropathic Pain Symptom Inventory: development and validation of a new algorithm. Pain. 2021;162:1038-46. https://doi.org/10.1097/j. pain.000000000000213.

38. Schmelz M. What can we learn from the failure of quantitative sensory testing? Pain. 2021;162:663-4. https://doi.org/10.1097/j. pain.0000000000002059.

39. Serpell M, Ratcliffe S, Hovorka J, Schofield M, Taylor L, Lauder $\mathrm{H}$, et al. A double-blind, randomized, placebo-controlled, parallel group study of THC/CBD spray in peripheral neuropathic pain treatment. Eur J Pain. 2014;18:999-1012.

40. Feingold D, Goor-Aryeh I, Bril S, Delayahu Y, Lev-Ran S. Problematic use of prescription opioids and medicinal cannabis among patients suffering from chronic pain. Pain Med. 2017;18:294-306. https://doi.org/10.1093/pm/pnw134.

41. Campbell G, Bruno R, Lintzeris N, Cohen M, Nielsen S, Hall $\mathrm{W}$, et al. Defining problematic pharmaceutical opioid use among 
people prescribed opioids for chronic noncancer pain: do different measures identify the same patients? Pain. 2016;157:1489-98. https://doi.org/10.1097/j.pain.0000000000000548.

42. Elander J, Lusher J, Bevan D, Telfer P. Pain management and symptoms of substance dependence among patients with sickle cell disease. Soc Sci Med. 2003;57:1683-96. https://doi.org/10. 1016/s0277-9536(02)00553-1.

43. Schmidt-Wolf G, Cremer-Schaeffer P. Begleiterhebung zur Anwendung von Cannabisarzneimitteln in Deutschland: Zwischenauswertung [Interim analysis of the survey accompanying insurance-covered prescriptions of cannabis-based medicines in Germany]. Bundesgesundheitsblatt Gesundheitsforschung Gesundheitsschutz. 2019;62:845-54. https://doi.org/10.1007/ s00103-019-02968-0.

44. Mohiuddin M, Blyth FM, Degenhardt L, Di Forti M, Eccleston C, Haroutounian S, et al. General risks of harm with cannabinoids, cannabis, and cannabis-based medicine possibly relevant to patients receiving these for pain management: an overview of systematic reviews. Pain. 2021;162(Suppl. 1):S80-96. https://doi. org/10.1097/j.pain.0000000000002000.

45. Moore RA, Fisher E, Finn DP, Finnerup NB, Gilron I, Haroutounian S, et al. Cannabinoids, cannabis, and cannabis-based medicines for pain management: an overview of systematic reviews.
Pain. 2021;162(Suppl. 1):S67-79. https://doi.org/10.1097/j.pain. 0000000000001941.

46. Fitzcharles MA, Niaki OZ, Häuser W, Hazlewood G, Canadian Rheumatology Association. Position statement: a pragmatic approach for medical cannabis and patients with rheumatic diseases. J Rheumatolol. 2019;46:532-8. https://doi.org/10.3899/ jrheum. 181120.

47. Häuser W, Morlion B, Vowles KE, Bannister K, Buchser E, Casale $\mathrm{R}$, et al. European clinical practice recommendations on opioids for chronic noncancer pain. Part 1: role of opioids in the management of chronic noncancer pain. Eur J Pain. 2021;25:949-68. https://doi.org/10.1002/ejp.1736.

48. Almog S, Aharon-Peretz J, Vulfsons S, Ogintz M, Abalia H, Lupo $\mathrm{T}$, et al. The pharmacokinetics, efficacy, and safety of a novel selective-dose cannabis inhaler in patients with chronic pain: a randomized, double-blinded, placebo-controlled trial. Eur J Pain. 2020;24:1505-16. https://doi.org/10.1002/ejp.1605.

49. Office of Medicinal Cannabis. Patient's guide medicinale cannabis. Available from: https://english.cannabisbureau.nl/medicinalcannabis/documents/circulars/2018/02/20/patients-guide-medic inal-cannabis. Accessed 21 June 2021. 